\title{
Coping and sickness absence
}

\author{
Willem van Rhenen • Wilmar B. Schaufeli • \\ Frank J. H. van Dijk · Roland W. B. Blonk
}

Received: 24 October 2006 / Accepted: 11 July 2007 / Published online: 16 August 2007

(C) Springer-Verlag 2007

\begin{abstract}
Objectives The aim of this study is to examine the role of coping styles in sickness absence. In line with findings that contrast the reactive-passive focused strategies, problemsolving strategies are generally associated with positive results in terms of well-being and overall health outcomes; our hypothesis is that such strategies are positively related to a low frequency of sickness absence and with short lengths (total number of days absent) and durations (mean duration per spell).

Methods Using a prospective design, employees' $(N=$ 3,628 ) responses on a self-report coping inventory are used to predict future registered sickness absence (i.e. frequency, length, duration, and median time before the onset of a new sick leave period).

Results and conclusions In accordance with our hypothesis, and after adjustment for potential confounders, employees with an active problem-solving coping strategy are less
\end{abstract}

W. van Rhenen · F. J. H. van Dijk

Academic Medical Center, Coronel Institute,

University of Amsterdam, Meibergdreef 9,

1105 AZ Amsterdam, The Netherlands

W. van Rhenen $(\square)$

Department of Occupational Health Services,

ArboNed Utrecht, Zwarte Woud 10,

3524 SJ Utrecht, The Netherlands

e-mail: willem.van.rhenen@ arboned.nl

W. B. Schaufeli $\cdot$ R. W. B. Blonk

Department of psychology and Research Institute of Psychology and Health, Utrecht University,

Heidelberglaan 1, 3584 CS Utrecht, The Netherlands

R. W. B. Blonk

TNO Work and Employment, Polarisavenue 151,

2132 JJ Hoofddorp, The Netherlands likely to drop out because of sickness absence in terms of frequency, length (longer than 14 days), and duration (more than 7 days) of sickness absence. This positive effect is observed in the case of seeking social support only for the duration of sickness absence and in the case of palliative reaction only for the length and frequency of absence. In contrast, an avoidant coping style, representing a reactivepassive strategy, increases the likelihood of frequent absences significantly, as well as the length and duration of sickness absence. Expression of emotions, representing another reactive-passive strategy, has no effect on future sickness absenteeism. The median time before the onset of a new episode of absenteeism is significantly extended for active problem-solving and reduced for avoidance and for a palliative response.

The results of the present study support the notion that problem-solving coping and reactive-passive strategies are inextricably connected to frequency, duration, length and onset of sickness absence. Especially, active problem-solving decreases the chance of future sickness absence.

Keywords Coping - UCL · Sickness absence $\cdot$ Duration · Length $\cdot$ Frequency

\section{Introduction}

A strong association exists between ill health and sickness absence, particularly for long absence spells (Marmot et al. 1995; Hensing et al. 1997). However, the decision of an employee to go on sick leave or to stay at work is not just the result of his or her (ill) health status alone (Aronsson et al. 2000; Rosvold and Bjertness 2001; Sandanger et al. 2000; Whitaker 2001; Anonymous 1979; Johansson and Lundberg 2004) but depends also on a number of 
demographic, social, and economic determinants (Johansson and Lundberg 2004; Voss et al. 2001; Eshoj et al. 2001). For instance, age (Sandanger et al. 2000), gender (Evans and Steptoe 2002), marriage (Mastekaasa 2000), level of education (Eshoj et al. 2001), salary (Chevalier et al. 1987), and sickness absence history (Landstad et al. 2001) are known to be associated with sickness absence behaviour. In addition, the way the individual deals with stressful situations (at work) is likely to affect his or her decision to report ill. In this article we focus on the role of this kind of socalled employee coping behaviour.

The relationship between coping and illness behaviour has been a major research focus over the past two decades (Somerfield and McCrae 2000). A variety of conceptual coping-frameworks have been proposed and numerous measures have been developed to assess ways of coping (McWilliams et al. 2003). Pioneering work in the field of coping has been carried out by Folkman and Lazarus (1980) who define coping as "the cognitive and behavioral efforts made to master, tolerate, or reduce external and internal demands and conflicts among them". In their opinion, coping has to be considered as a behaviour that is primarily determined by environmental demands, that is, coping is an individual response to a stressful environment. In contrast, other scholars (Holahan et al. 1996; Moos and Holahan 2003) consider coping primarily as a trait or as a resource. The former refers to a relatively stable personal characteristic: that is, similar coping strategies are used across a wide variety of situations (Parker and Endler 1992; Carver and Scheier 1994). The latter refers to the use of particular social and personal characteristics: that is, personal resources on which the individual may draw upon when dealing with stressful situations (Pearlin and Schooler 1978). This trait- or dispositional approach of coping implies a stable coping style or a coping resource regularly used.

As early as four decades ago, Kahn et al. (1964) distinguished between two general coping strategies: problemsolving strategies and reactive-passive strategies. Their idea of two general coping strategies has been worked out by Lazarus and Folkman (1984) in what nowadays is probably the most popular and widely accepted conceptualization of coping behaviour. Problem solving-coping refers to active strategies that are directly targeted at solving the problem at hand, whereas reactive-passive focused coping refers to those strategies that reduce the negative emotions that are evoked by the stressful situation (Elfering et al. 2005).

Much research on coping strategies reveals that both reactive-passive strategies and avoidance strategies result in psychological and physical symptoms (Terry et al. 1996; Pisarski et al. 1998; Penley et al. 2002), whereas active, problem-solving coping generally has a positive impact on well-being and overall health outcomes (Penley et al. 2002). However, in their recent review, Austenfeld and Stanton
(2004) criticized this popular and almost generally accepted conclusion. They identified over a hundred articles examining the relationship between reactive-passive coping and adjustment (Stanton et al. 2002b) and found that hardly any of the coping instruments contained the same set of coping strategies, which made it practically impossible to aggregate the findings. Furthermore, the association between reactivepassive strategies and psychological and physical symptoms appeared to be related to the way these strategies had been operationalized (Stanton et al. 2002a). It appeared that corruption of the original coping items as well as the use of item formulations that include the expression of emotional distress or self-deprecation result in spurious correlations.

Studies on coping and sickness absence are scarce. Kristensen (1991) was among the first to investigate this relationship and he asserted that sickness absence itself should be regarded as coping behaviour reflecting the individual's perception of health or illness. Sickness absence itself, in his opinion, is a functional coping strategy, used by employees to reduce work-related strain by avoiding the workplace and thus creating for themselves the opportunity for recuperation. Kristensen was one of the first not to primarily focus on determinants of sickness absence, but rather tried to understand sickness absence from a coping perspective. By doing so, he went beyond existing concepts of coping by considering sickness absence "a type of coping behaviour" (Kristensen 1991). As he stated: "sickness absence can well be a rational coping behaviour seen in the light of a person's wish to maintain his/her health and working capacity: as such it is the opposite of withdrawal behaviour". Clearly, this approach differs from considering coping as a personality trait or resource.

In the present study, coping is conceptualized and measured as a trait or disposition i.e. it is assumed that individuals tend to use rather similar coping strategies across a wide variety of situations. The Utrecht coping list (UCL) (Schreurs et al. 1993) was selected to assess the employees' coping style. This well-validated self-report questionnaire is the most widely used coping inventory in the Netherlands, both in research and in practice (Schreurs et al. 1993; Schaufeli and Van Dierendonck 1992; Norberg et al. 2005; Buitenhuis et al. 2003). Like the COPE questionnaire of Carver et al. (1989), the UCL asks individuals how they deal with stressful situations; that is, how often they engage in various exertions encountering problems or unpleasant occurrences. The UCL distinguishes between five coping styles that can be grouped together into two higher-order coping styles: active, problem-solving and a reactive-passive style (Schaufeli and Van Dierendonck 1992). Hence, the UCL offers the possibility to investigate employees' coping styles at a more detailed level, at the same time taking into account the conceptual distinction between problem-solving and reactive-passive coping. 
Sickness absence has been measured in terms of frequency, (total) length of sickness absence, (mean) duration of sickness absence spells as well as by the sickness absence free interval. These sickness absence measures are defined in accordance with recommendations of Hensing et al. (1998) who pleaded for a more standardized international description of sickness absence measures. In their literature review, Hensing et al. pointed out the multi-interpretability of sick leave indicators and recommended basic measures to encompass the full spectrum of the sickness absence phenomenon to make studies more accessible for international comparisons. Recently, a study by Landstad et al. (2001) confirmed this line of reasoning by concluding that different forms of absenteeism need to be studied simultaneously, in order to distinguish changes in sickness absence pattern correctly.

In summary then, the aim of the study is to examine the role of coping styles in sickness absence. Based on the fact that, contrary to reactive-passive strategies, problem-solving strategies are generally associated with positive results in terms of well-being and overall health outcomes, our hypothesis is that such strategies are positively related to a low frequency of sickness absence and with short lengths and durations. Reactive-passive strategies, on the other hand, are not expected to be related to sickness absence.

\section{Subjects and methods}

Study population and participants

Participants were employees of a large Dutch telecom company. An occupational health survey was sent to all 7,522 employees, including an assessment of coping strategies (response rate $51 \% ; N=3,852$ ). Sickness absence of the participants was followed up for 1 year after the survey. Due to missing sickness absence data, the sample was reduced to 3,628 employees [3,302 men (mean age 44.7 years, $\mathrm{SD}=7.5$ ) and 311 women (mean age 39.7 years, $\mathrm{SD}=8.7)$ ]. A description of the sample is shown in Table 1. During the first quarter after the start of the study, $64 \%$ of the participants have not been absent because of sickness, whereas, $7 \%$ of the participants have been absent for more than 14 days (length).

Compared to non-participants, participants were predominantly male, older, better paid, and were less absent for sickness (see Table 2).

\section{Measures}

\section{Coping style}

We assessed the coping strategy of the participants using the shortened 19-item version of the original 30-item
Table 1 Demographics and absenteeism of participants

\begin{tabular}{ll}
\hline Variable & \\
\hline Male & $91 \%$ \\
Age, mean (SD) (min-max) years & 44.2 (7.7) (22-63) \\
Marital status & \\
Married or cohabiting & $79 \%$ \\
Single & $17 \%$ \\
Divorced or separated & $4 \%$ \\
Educational level & \\
Lower vocational education & $27 \%$ \\
Intermediate vocational education & $50 \%$ \\
Higher vocational education and university & $21 \%$ \\
Missing/something else & $2 \%$ \\
Working years present job & \\
1 year & $30 \%$ \\
$>1-5$ year & $43 \%$ \\
$>5-10$ year & $14 \%$ \\
$>10$ year & $14 \%$ \\
Sickness absence first quarter & \\
0 days & \\
$1-7$ days & $64 \%$ \\
>14 days & $22 \%$ \\
Function & $6 \%$ \\
Blue collar (executive) & $7 \%$ \\
Office workers (administrative) & \\
Supervisors & \\
Consultants & $6 \%$ \\
Managerial staff & $16 \%$ \\
\hline & \\
\hline
\end{tabular}

Utrecht Coping List (UCL) (Schreurs et al. 1993). This questionnaire was designed to measure the coping strategies people use in stressful situations, either life events or daily hassles. Each item is rated on a four-point Likert scale ranging from one (never) to four (very often).

The UCL includes five dimensions; (1) active problemfocusing (five items, e.g. thinking of different possibilities to solve a problem), (2) seeking social support (5 five items, e.g. seeking comfort and sympathy), (3) palliative reaction pattern (four items, e.g. looking for distraction), (4) avoidance behavior (three items, e.g. complying to avoid problematic situations) and (5) expression of emotions (two items, e.g. showing frustrations). The first three coping styles were found to cluster into a second-order active problem-solving factor, whereas both final styles clustered into a reactive-passive factor (Schaufeli and Van Dierendonck 1992). According to the test manual, the internal consistencies as well as the test-retest reliability are satisfactory (Schreurs et al. 1993). In order to assess the factorial validity of the shortened UCL in our employee sample, a confirmative factor analysis was carried out. 
Table 2 Demographics and absenteeism of participants and non-participants

\begin{tabular}{lllll}
\hline & $\begin{array}{l}\text { Participants } \\
(n=3,628)\end{array}$ & $\begin{array}{l}\text { Non-participants } \\
(n=3,670)\end{array}$ & $T$ Test $\mathrm{p}$ & $\chi^{2} \mathrm{p}$ \\
\hline Gender (\%women) & 8.6 & 14.1 & 0.000 & 0.000 \\
Age, mean (SD) in years & $44.2(7.7)$ & $40.7(9.3)$ & & 0.000 \\
Salary (\%) & 40.6 & 53.2 & \\
Low & 42.9 & 33.3 & \\
Medium & 16.5 & 13.5 & \\
High & $14.9(39.9)$ & $22.9(59.3)$ & 0.000 & \\
Absenteeism & $1.20(1.31)$ & $1.31(1.46)$ & 0.000 \\
Length mean (SD) days & & & \\
Frequency & & & \\
\hline
\end{tabular}

\section{Sickness absence}

Sickness absence data were taken from the sickness absence records of the employees filed in the database of ArboNed, an occupational health service (OHS) serving the telecom company. All spells of absence for medical reasons were centrally reported and registered by the executive manager of the company. Absence spells longer than 2 weeks were verified by an occupational physician by inviting the employee on sick leave for an interview. Therefore, the validity of the absence data is assumed to be high.

Measures used are (1) (total) length of sickness absence in current and new spells during the study period (1 year) per sick listed person (i.e. total number of days absent) (2) frequency of sickness absence (new sick-leave spells during the study period (1 year) and (3) (mean) duration of sickness absence (sick-leave days in new spells during the study period (1 year) per spell). The duration of sickness absence is classified into more or less than 7 days. In our sample, short-term sickness (less than 7 days) accounts for $75 \%$ of the absences and mainly represents minor ailments. Finally, we assessed the median time before the onset of a new sick leave period after the occupational health survey.

\section{Statistical analysis}

Confirmatory factor analysis (CFA), using the AMOS 5 software program (Arbuckle 2003) was used to test the fit of two competing models: M1 that assumes that all 19 items load on one general coping factor, and M2 that assumes that the items load on the five hypothesized correlated factors. Maximum likelihood estimation methods were used and the input for each analysis was the covariance matrix of the items. The goodness-of-fit of both models was evaluated using the $\chi^{2}$ goodness-of-fit statistic and the root mean square error of approximation (RMSEA). However, $\chi^{2}$ is sensitive to sample size so that the probability of rejecting a hypothesized model increases when sample size increases, even if the difference between the fitted model and the "true" underlying model is very small. To overcome this problem, the computation of relative goodness-of-fit indices is strongly recommended (Bentler 1990). Three relative goodness-of-fit indices were computed: the normed fit index (NFI), the non-normed fit index (NNFI) and the comparative fit index (CFI). The latter is particularly recommended for model comparison purposes (Goffin 1993). For all relative fit-indices, as a rule of thumb, values greater than 0.90 are considered as indicating a good fit (Byrne, 2001, pp. 79-88), whereas values smaller than .08 for RMSEA indicate acceptable fit (Cudeck and Browne 1993). Next, Cronbach alphas were calculated for the UCLsubscales.

In a next step, scale scores for different coping strategies were calculated and transformed into scale scores ranging from 0 to 100 . Finally, tertiles of the distribution of the 0-100 scale scores were used to distinguish between low-, medium- and high levels of the coping strategies.

To examine the relationship between coping and sickness absence, odds ratios and corresponding 95\% confidence intervals were calculated using logistic regression analysis.

Stepwise multiple logistic regression analysis was used to study the (confounding) influence of sociodemographic factors and other determinants on the relationship between coping and sickness absence. The magnitude of the (confounding) effects was assessed by calculating the proportion of the excess risk (OR minus 1.0) explained when fitting these terms in the model.

Finally, the period between the health surveillance and the onset of a new period of absenteeism was evaluated using survival analysis. Since we wish to estimate the probability of absenteeism at a designated time interval (conditional probability) the Kaplan-Meier methodology (Kaplan and Meier 1958) has been applied. With this statistical technique, means, medians and confidence intervals of the 'survival' (in this study: the onset of absenteeism) are calculated without making assumptions about the survival distribution. 


\section{Results}

\section{UCL factor structure}

As can be seen in Table 3, confirmatory factor analysis (EFA) corroborated the underlying five-factor structure of the short form of the UCL. More particularly, all fit-indices of M2-the hypothesized model with five correlated factors-sufficed their respective criteria, except NNFI that approached its criterion of 0.90 . The mean correlation between the five factors was 0.24 , ranging from -0.04 to 0.45. Moreover, the fit of M2 was superior to that of M1 that assumed that all items load on one undifferentiated coping factor $\left(\Delta \chi^{2}=10146.22 ; d f=10 ; P<0.001\right)$. Hence the factorial validity of the UCL-15 was demonstrated.

The Cronbach alphas for the subscales avoidance behaviour, expression of emotions, seeking social support, active problem-focusing and palliative reaction in this study were $0.67,0.65,0.76,0.81$ and 0.68 , respectively. Although some values are slightly below 0.70 , which is recommended for established scales, all values are well above 0.60 , which is deemed satisfactory for newly developed scales (Nunnaly and Bernstein 1994).

\section{Sickness absence and demographics}

As can be seen from Table 4, length (total number of days absent) and duration (mean duration per spell) of sickness absence are associated with gender (i.e. women), being divorced or single, having an intermediate or lower education, a shorter period working in the present (current) job, lower salary, higher age, and a history of sickness absence both for length and frequency. Likewise, a higher frequency of sickness absence was associated with gender (i.e. women), being divorced, an intermediate salary and a history of sickness absence both for length and frequency. In our sample there is no association between absence frequency and level of education, the period working in the current job, or age.

Sickness absence and ways of coping

As displayed in Table 5, a greater length (total number of days) of sickness absence is predicted by low- or medium- active problem-focusing, avoidance behaviour and a medium- or high palliative reaction. The frequency and the duration of sickness absence are associated in a similar way, however, the latter showing a relation with low seeking social support rather than a palliative reaction. Table $5 \mathrm{~b}$ summarizes the significant associations between various sickness absence measures and ways of coping (Table 5). It can be seen from this table that the crude ORs of the active and avoidant coping styles show the most consistent patterns of associations across all sickness absence measures.

Sickness absence, and demographics and ways of coping

Of course, the question arises whether or not the association between coping and sickness absence could be explained by previous sickness absence and by demographics. Therefore, Table 6 displays the ORs for the three sickness absence measures with coping strategies after adjustment for previous sickness absence and the demographics mentioned in Table 4.

\section{Length}

Adjustment for sickness absence history increases the excess risk to be absent for more than 14 days in one year by $22 \%$ for active problem-focusing (thus, sickness absence history reduces the effect of active coping), while reducing it by 25 and 33\% for palliative reaction and avoidance coping, respectively. After adjustment for gender and sickness absence history, the excess risk for length in addition to palliative reaction and sickness absence history decreases by $17 \%$.

The excess risk for length adjusted for salary in addition to active problem-focusing, sickness absence history and gender increases by $18 \%$. In summary, adjusted for several confounding variables, the length of sickness absence is effectively influenced by active problem-focusing and palliative reaction.

\section{Frequency}

Adjustment for sickness absence history barely minimizes the risk for frequency by coping considering active problem-focusing and avoidance behaviour. For palliative

Table 3 Fit indices of one-factor (M1) and two-factor (M2) models of coping (UCL-19)

\begin{tabular}{lrlllllll}
\hline Model & $\chi^{2}$ & $d f$ & GFI & AGFI & RMSEA & NFI & NNFI & CFI \\
\hline M1 & 1030.29 & 152 & 0.69 & 0.61 & 0.14 & 0.43 & 0.36 \\
M2 & 184.07 & 142 & $0 . .95$ & 0.93 & 0.06 & 0.90 & 0.88 \\
Null model & 17976.60 & 171 & 0.54 & 0.49 & 0.17 & - & - & - \\
\hline
\end{tabular}

GFI goodness of fit index, $A G F I$ adjusted goodness of fit index, RMSEA root mean square estimate of approximation, NFI normed fit index, NNFI non-normed fit index, $C F I$ comparative fit index; all $\chi^{2}, P<0.001$ 
Table 4 Associations of demographics and sickness absence $n$ ranges between 3,575-3,606 due to missing values

\begin{tabular}{|c|c|c|c|c|c|c|}
\hline & \multicolumn{6}{|c|}{ Sickness absence } \\
\hline & \multicolumn{2}{|c|}{ Length $>14$ days } & \multicolumn{2}{|c|}{ Duration $>7$ days } & \multicolumn{2}{|c|}{ Frequency $>2 x$} \\
\hline & OR & $95 \% \mathrm{CI}$ & OR & $95 \% \mathrm{CI}$ & OR & $95 \% \mathrm{CI}$ \\
\hline \multicolumn{7}{|l|}{ Gender } \\
\hline Woman & 1.00 & & 1.00 & & 1.00 & \\
\hline Man & 0.49 & $0.38-0.62$ & 0.66 & $0.51-0.85$ & 0.42 & $0.32-0.55$ \\
\hline \multicolumn{7}{|l|}{ Married } \\
\hline Married & 1.00 & & 1.00 & & 1.00 & \\
\hline Single & 0.83 & $0.66-1.03$ & 1.25 & $1.03-1.52$ & 1.05 & $0.82-1.36$ \\
\hline Divorced & 1.73 & $1.24-2.41$ & 2.18 & $0.54-8.81$ & 1.54 & $1.04-2.28$ \\
\hline \multicolumn{7}{|l|}{ Education } \\
\hline University & 1.00 & & 1.00 & & 1.00 & \\
\hline Higher vocational education & 0.88 & $0.59-1.33$ & 1.13 & $0.72-1.78$ & 1.00 & $0.63-1.59$ \\
\hline Interm. vocational education & 1.42 & $1.00-2.00$ & 1.93 & $1.30-2.86$ & 1.16 & $0.78-1.72$ \\
\hline Lower vocational education & 2.07 & $1.45-2.96$ & 2.84 & $1.90-4.24$ & 1.30 & $0.86-1.96$ \\
\hline \multicolumn{7}{|l|}{ Present (current) job } \\
\hline$>10$ years & 1.00 & & 1.00 & & 1.00 & \\
\hline $5-10$ years & 0.98 & $0.74-1.28$ & 1.02 & $0.78-1.34$ & 1.04 & $0.74-1.46$ \\
\hline$<5$ years & 0.67 & $0.54-0.83$ & 0.61 & $0.49-0.76$ & 0.91 & $0.69-1.19$ \\
\hline \multicolumn{7}{|l|}{ Salary } \\
\hline Low 4-6 & 1.00 & & 1.00 & & 1.00 & \\
\hline Intermediate 7-9 & 0.50 & $0.43-0.60$ & 0.49 & $0.41-0.59$ & 1.71 & $1.28-2.28$ \\
\hline High $>9$ & 0.38 & $0.30-0.49$ & 0.33 & $0.25-0.44$ & 1.07 & $0.79-1.44$ \\
\hline \multicolumn{7}{|l|}{ Age } \\
\hline$<35$ years & 1.00 & & 1.00 & & 1.00 & \\
\hline $35-45$ years & 1.38 & $1.07-1.78$ & 1.54 & $1.17-2.02$ & 1.20 & $0.90-1.60$ \\
\hline$>45$ years & 1.48 & $1.16-1.88$ & 1.79 & $1.38-2.32$ & 0.93 & $0.70-1.22$ \\
\hline \multicolumn{7}{|c|}{ History sickness absence in days (length) 1 year before } \\
\hline 0 & 1.00 & & 1.00 & & 1.00 & \\
\hline $1-7$ & 1.57 & $1.24-2.01$ & 1.19 & $0.95-1.50$ & 3.07 & $2.18-4.31$ \\
\hline $8-14$ & 3.68 & $2.85-4.74$ & 2.65 & $2.08-3.39$ & 6.30 & $4.44-8.95$ \\
\hline$>14$ & 9.72 & $7.75-12.2$ & 4.26 & $3.43-5.29$ & 12.9 & $9.40-17.8$ \\
\hline \multicolumn{7}{|l|}{ History frequency ( 1 year before) } \\
\hline $0 \mathrm{x}$ & 1.00 & & 1.00 & & 1.00 & \\
\hline $1-2 x$ & 2.77 & $2.26-3.41$ & 1.91 & $1.58-2.32$ & 3.96 & $2.91-5.40$ \\
\hline$>2 x$ & 8.66 & $6.81-11.02$ & 3.83 & $3.04-4.84$ & 17.47 & $12.6-24.2$ \\
\hline
\end{tabular}

coping, the reduction for the excess risk amounts to $51 \%$. When adjusted for gender, in addition to sickness absence history, the risk of high frequency in association with palliative reaction reduces by another $16 \%$. In sum, adjusted for several confounding variables the frequency of sickness absence is effectively influenced by active problem-focusing, avoidance behaviour and expression of emotions.

\section{Duration}

Adjustment for sickness absence history reduces the excess risk of active problem focusing by $24 \%$, of seeking social support and palliative reaction by $16 \%$, and of avoidance behaviour by $12 \%$. Adjustment for gender in association with seeking social support affects the excess risk of duration by $16 \%$. In summary, adjusted for several confounding variables, the duration of sickness absence is effectively influenced by active problem focusing, avoidance behaviour and seeking social support.

\section{Effects on the onset of a new period of absenteeism}

During the first year, the median time before the onset of a new episode of absenteeism is significantly shorter for those low in active problem-focusing, high in avoidance, and high in a palliative response. For the two remaining 
Table 5 Associations of coping and sickness absence

\begin{tabular}{|c|c|c|c|c|c|c|c|}
\hline & & \multicolumn{6}{|c|}{ Sickness absence } \\
\hline & & \multicolumn{2}{|c|}{ Length $>14$ days } & \multicolumn{2}{|c|}{ Duration $>7$ days } & \multicolumn{2}{|c|}{ Frequency $>2 x$} \\
\hline & & OR & $95 \% \mathrm{CI}$ & OR & $95 \% \mathrm{CI}$ & OR & $95 \% \mathrm{CI}$ \\
\hline \multirow[t]{12}{*}{ Problem-solving } & \multicolumn{7}{|c|}{ Active problem-focusing } \\
\hline & Low & 1.00 & & 1.00 & & 1.00 & \\
\hline & Medium & 0.84 & $0.70-0.99$ & 0.83 & $0.69-0.99$ & 0.84 & $0.68-1.04$ \\
\hline & High & 0.61 & $0.49-0.75$ & 0.69 & $0.56-0.86$ & 0.78 & $0.53-0.87$ \\
\hline & \multicolumn{7}{|c|}{ Seeking social support } \\
\hline & Low & 1.00 & & 1.00 & & 1.00 & \\
\hline & Medium & 0.98 & $0.82-1.17$ & 0.97 & $0.80-1.16$ & 1.01 & $-0.80-1.26$ \\
\hline & High & 0.92 & $0.75-1.12$ & 0.81 & $0.66-0.99$ & 1.15 & $0.90-1.45$ \\
\hline & \multicolumn{7}{|c|}{ Palliative reaction } \\
\hline & Low & 1.00 & & 1.00 & & 1.00 & \\
\hline & Medium & 1.22 & $1.01-1.49$ & 1.15 & $0.94-1.41$ & 1.43 & $1.13-1.81$ \\
\hline & High & 1.33 & $1.11-1.59$ & 1.19 & $0.99-1.43$ & 1.40 & $1.12-1.74$ \\
\hline \multirow[t]{8}{*}{ Reactive-passive } & \multicolumn{7}{|c|}{ Avoidance behaviour } \\
\hline & Low & 1.00 & & 1.00 & & 1.00 & \\
\hline & Medium & 1.11 & $0.92-1.32$ & 1.14 & $0.94-1.37$ & 1.22 & $0.98-1.51$ \\
\hline & High & 1.35 & $1.10-1.65$ & 1.32 & $1.07-1.63$ & 1.39 & $1.09-1.67$ \\
\hline & \multicolumn{7}{|c|}{ Expression of emotions } \\
\hline & Low & 1.00 & & 1.00 & & 1.00 & \\
\hline & Medium & 1.05 & $0.87-1.28$ & 1.21 & $0.99-1.48$ & 0.91 & $0.72-1.14$ \\
\hline & High & 1.19 & $0.95-1.50$ & 1.13 & $0.89-1.44$ & 1.29 & $0.99-1.69$ \\
\hline
\end{tabular}

Summary table 5

\begin{tabular}{lllll}
\hline & & \multicolumn{2}{l}{ Sickness absence } & \\
Problem-solving & & Length & Duration & Frequency \\
& Active problem-focusing & $\mathrm{X}$ & $\mathrm{X}$ & $\mathrm{X}$ \\
& Seeking social support & - & $\mathrm{X}$ & - \\
& Palliative reaction & $\mathrm{X}$ & - & $\mathrm{X}$ \\
Reactive-passive & Avoidance behaviour & $\mathrm{X}$ & $\mathrm{X}$ & $\mathrm{X}$ \\
& Expression of emotions & - & - & - \\
\hline
\end{tabular}

coping styles, no significant results were found (Table 7). This means that employees who are used to solving problems actively instead of avoiding problems or engaging in alternative behaviours, enter sick leave later, the next time.

\section{Discussion}

In accordance with our hypothesis, and after adjustment for potential confounders, employees with an active problemsolving coping strategy are less likely to drop out because of sickness absence in terms of frequency, length (total number of days absent, longer than 14 days), and duration (mean duration per spell, more than 7 days) of sickness absence. This positive effect is observed in the case of 'seeking social support' only for duration of sickness absence, and in the case of 'palliative reaction' only for length and frequency of sickness absence. In contrast, an avoidant coping style, representing a reactive-passive strategy, significantly increases the likelihood of frequent absences, as well as the duration of sickness absence. Expression of emotions, representing another reactive-passive strategy, has no effect on sickness absence. The median time before the onset of a new episode of absenteeism, finally, is significantly extended for active problem-solving and reduced for avoidance and for a palliative response.

In summary, we conclude that in accordance with our hypothesis, a problem-solving coping strategy, in contrast to a reactive-passive coping strategy, significantly reduces sickness absence. This result seems to corroborate other research findings that showed that problem-solving coping is associated with well being and overall health outcomes (Kohn 1996). On the other hand, our results are at odds with research findings that document a positive relationship 


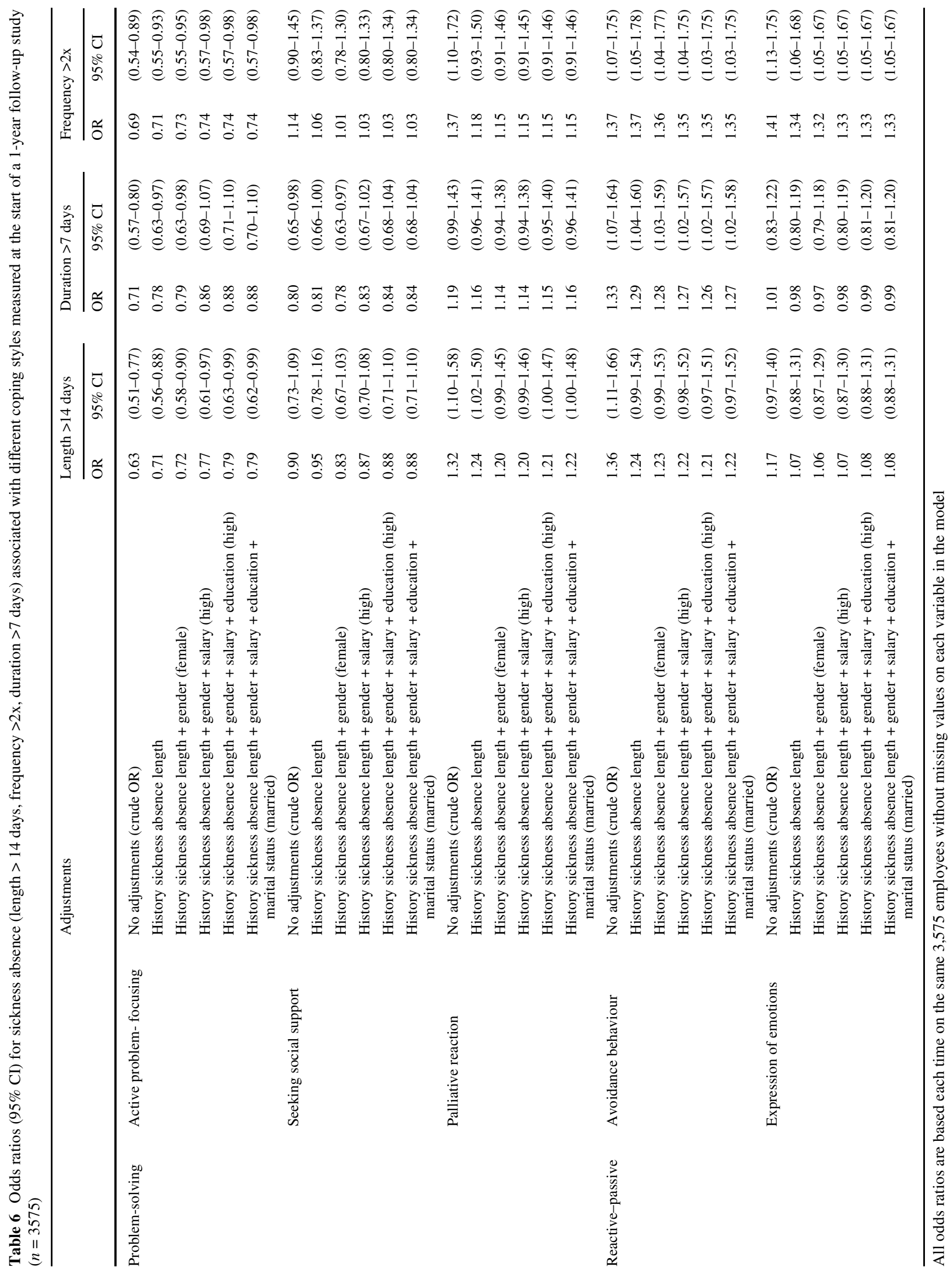


Table 7 Kaplan-Meier: the relation between different coping styles and the onset of absenteeism in the year after coping assessment

\begin{tabular}{|c|c|c|c|c|c|c|c|}
\hline & & \multicolumn{6}{|l|}{ Log rank } \\
\hline & & Median (days) & SE & $95 \% \mathrm{CI}$ & Stat & $d f$ & Sign \\
\hline \multirow[t]{12}{*}{ Problem-solving } & \multicolumn{7}{|c|}{ Active problem-focusing } \\
\hline & Low & 152 & 7 & $137-167$ & & & \\
\hline & Medium & 170 & 13 & $145-195$ & & & \\
\hline & High & 176 & 14 & 149-203 & 9.44 & 2 & 0.01 \\
\hline & \multicolumn{7}{|c|}{ Seeking social support } \\
\hline & Low & 168 & 10 & $148-188$ & & & \\
\hline & Medium & 165 & 8 & $148-182$ & & & \\
\hline & High & 155 & 17 & $122-188$ & 0.45 & 2 & 0.80 \\
\hline & \multicolumn{7}{|c|}{ Palliative reaction } \\
\hline & Low & 182 & 14 & 155-209 & & & \\
\hline & medium & 155 & 12 & $132-178$ & & & \\
\hline & High & 146 & 7 & $131-161$ & 13.65 & 2 & 0.00 \\
\hline \multirow[t]{8}{*}{ Reactive-passive } & \multicolumn{7}{|c|}{ Avoidance behaviour } \\
\hline & Low & 182 & 11 & $160-204$ & & & \\
\hline & Medium & 151 & 11 & $130-172$ & & & \\
\hline & High & 144 & 10 & $125-163$ & 14.6 & 2 & 0.00 \\
\hline & \multicolumn{7}{|c|}{ Expression of emotion } \\
\hline & low & 165 & 10 & $146-184$ & & & \\
\hline & Medium & 167 & 11 & $145-189$ & & & \\
\hline & High & 156 & 12 & $133-179$ & 0.98 & 2 & 0.61 \\
\hline
\end{tabular}

between reactive-passive coping and health (Austenfeld and Statton 2004; Coyne and Racioppo 2000). Austenfeld and Statton (2004) have argued that the negative effect of reactive-passive coping on health may partly be attributed to the operationalizaton of this construct, and therefore recommended a clear description of the reactive-passive coping items used. The idea is that reactive-passive coping can be separated into two factors, namely emotional expression and emotional processing (Lazarus 1993). The former factor is an active attempt to acknowledge, explore meanings or come to an understanding of one's emotions. Items measuring emotional processing, however, focus on the acknowledgement of emotions, the validness and importance of feelings, the delving into the feelings. Especially, emotional processing has a positive association with health, although how the influencing occurs is still unclear. The items that tap reactive-passive coping in the UCL refer to the expression of emotions and not to their processing. This probably explains the indifferent and negative effect on sickness absence by 'emotional expression' and 'avoidance behaviour', respectively. A second possible explanation can be that reactive-passive strategies have a positive relationship to health but not necessarily with sickness absence.

Our study partly refutes the assumption of Kristensen (1991) that sickness absence is a coping strategy by itself. Kristensen claimed that employees who use sickness absence as a coping strategy would experience less work- related strain, especially in jobs with poor decision latitude. Accordingly, because they are no longer exposed to their stressful jobs, employees would recuperate during sickness absence, especially in the case of psychosomatic symptoms. In our study, sickness absence history that can be considered a proxy of the coping strategy of sickness absence had only a minor impact on sickness absence given a general coping style. And although the effect is less strong, the measured coping strategies of the UCL still have an effect on sickness absence.

The favourable outcome of problem-solving coping in relation to sickness absence can be attributed to being engaged in active transactions between person and environment with the aim of alleviating stress-inducing situations (Lazarus 1993; Huizink et al. 2002; Roesch and Weiner 2001). Efforts to remove the stressor, gathering information, and finding possible solutions for the problems are a few examples. In general, these strategies are associated with self-confidence and perceived control, and are observed in individuals who are persistent and assertive, self-efficacious, and less anxious and depressed (Heppner 1988; Heppner and Baker 1997).

Two factors in the evaluation of problem-solving coping should be commented upon. Men are believed to be more likely to confront a problem with active coping, whereas women are believed to exhibit a more reactive-passive response (Pearlin and Schooler 1978; Hamilton and Fagot 
1998). For instance, a meta-analysis of Tamres et al. (2002) showed that compared to men, women are more likely to use indirect strategies that involve verbal expression or to seek emotional support. Huizink et al. (2002), however, argue that the presumed effectiveness of problem-solving strategies is based on the assumption that male-gender role behaviour is superior. She suggests that studies, as a result of gender bias, have failed to identify other styles of coping as potentially effective. In our study, however, considering several styles, the adjustment for gender barely affects the influence of coping on sickness absence measures.

Another complicating factor in the evaluation of the effectiveness of problem-solving coping may be that reviewers group several distinct coping behaviours under this one single coping category in an effort to simplify the findings (Tamres et al. 2002). For instance, problem-solving coping may be composed of different behaviours. This is underscored by our finding that different problem-solving strategies have different outcomes on sickness absence. Seeking social support, for example, affects only duration (marginally), whereas active problem-focusing affects length, duration and frequency. The difference in outcome of different sickness absence measures in the case of seeking social support may be clarified by Stansfeld et al. (1997) who argues that social support may influence absence-related behaviour and encourage a person to take absence at a time of illness. Contrarily, one may postulate that social support also shortens sickness absence. Both postulations may result in the absence of a substantial effect.

To the authors' knowledge, this is the first study with four sick leave outcome measures in relation to coping that reveals a more comprehensive picture of changes in the sick leave pattern. In line with Isacsson et al. (1992), we can conclude that "adding more measures gives a more comprehensive picture of sickness absenteeism and of differences between groups". For instance, the present study demonstrates a relation between a palliative coping reaction and length of sickness absence in contrast with the duration of sick leave. Without the differential pattern for sickness absence, the differential effects of several coping strategies would remain invisible. Another, and perhaps even more important, argument to use different measures of sickness absence is the accessibility of this study for international comparisons in future research.

Finally, the multi-factorial aetiology of sickness absence requires discussion. Alexanderson (1998) pointed out that different disciplines and scientific traditions deal in different ways with absenteeism. In medical science, for instance, the focus of research is on occurrence, etiology and intervention, whereas the focus in medical sociology is on interacting factors within a pre-circumscribed model. She and other authors (Whitaker 2001; Alexanderson 1998), therefore categorized the many factors of sickness absence in three levels: macro/national level (Alexanderson 1995) (e.g. insurance systems), organizational level (Jeurissen and Nyklicek 2001; Vahtera et al. 1996) (e.g. job demands, resources) and individual level (e.g. gender, education). Recognizing this phenomenon, our analyses were adjusted for several known risk factors at the level of the individual. Since the present study was conducted in one Dutch company, the influence of organizational and socioeconomic factors was equally present in all groups and in this sense controlled for.

A strong point of our study is the detailed way in which sickness absence is assessed, using objective archival data. Thus far, relatively little attention has been paid to the implications of different quantitative measures of sickness absence. Moreover, a prospective design was used that allowed for predicting future sickness absenteeism.

A limitation of the study is the non-recurring measurement of coping in our study. Therefore, we cannot rule out the possibility that sickness absence might influence the way employees cope with stressful situations. Although, coping styles, as measured with the UCL, have proven to be relatively stable in time (Norberg et al. 2005), reversed causation cannot be ruled out.

A second limitation could be the Cronbach's alpha of some subscales of the UCL (slightly below the 0.70 ). However, the criterion of 0.70 is an arbitrary value that is not universally accepted as the minimum level of acceptability. As an example of the arbitrariness of this criterion, Nunnally (1967) mentioned that $\alpha$ s ranging from $0.50-0.60$ would be acceptable, but in the second edition of that book he suggests that 0.70 is the minimally acceptable valuewithout further justification (Nunnally 1978). Moreover, the minimally required degree of reliability is a function of the research purpose; for individual-level, diagnostic research $\alpha$ should be much higher than for the basic, grouplevel research reported in our study (Peterson 1994). Hence we used a minimum threshold for coefficient $\alpha$ of 0.65 as was recently proposed by De Vellis (2003).

In spite of these limitations, the results of the present study support the notion that problem-solving coping and reactive-passive strategies are inextricably connected with frequency, duration, length and onset of sickness absence. Especially 'active problem-focusing' decreases the chance of future sickness absence.

\section{References}

Alexanderson K (1995) Sickness absence in a Swedish county, with references to gender, occupation, pregnancy and parenthood. University of Linköping. Thesis/Dissertation

Alexanderson K (1998) Sickness absence: a review of performed studies with focused on levels of exposures and theories utilized. Scand J Soc Med 26:241-249 
Anonymous (1979) Sickness absence in hospital staff. Lancet 2(8155):1278-1279

Arbuckle JL (2003) AMOS 5.0 (Computer Software). SPSS, Chicago

Aronsson G, Gustafsson K, Dallner M (2000) Sick but yet at work. An empirical study of sickness presenteeism. J Epidemiol Community Health 54:502-509

Austenfeld JL, Stanton AL (2004) Coping through emotional approach: a new look at emotion, coping, and health-related outcomes. J Pers 72(6):1335-1363

Bentler PM (1990) Comparative fit indexes in structural equation models. Psychol Bull 107:238-246

Buitenhuis J, Spanjer J, Fidler V (2003) Recovery from acute whiplash: the role of coping styles. Spine 28(9):896-901

Byrne BM (2001) Structural equation modeling with AMOS: basic concepts, applications, and programming. Erlbaum, Mahwah

Carver CS, Scheier MF (1994) Situational coping and coping dispositions in a stressful transaction. J Pers Soc Psychol 66:184-195

Carver CS, Scheier MF, Weintraub JK (1989) Assessing coping strategies: a theoretically based approach. J Pers Social Psychol $56: 267-283$

Chevalier A, Luce D, Blanc C, Goldberg M (1987) Sickness absence at the French National Electric and Gas Company. Br J Ind Med 44(2):101-110

Coyne JC, Racioppo MW (2000) Never the twain shall meet? Closing the gap between coping research and clinical intervention research. Am Psychol 55:655-664

Cudeck R, Browne MW (1993) Alternative ways of assessing model fit. In: Bollen KA, Scott Long J (eds) Testing structural equation models. Sage, Newbury Park, pp 1-9

De Vellis RF (2003) Scale development: theory and applications, 2nd edn. Sage, Thousand Oaks

Elfering A, Grebner S, Semmer NK, Kaiser-Freiburghaus D, Ponte SL, Witschi I (2005) Chronic job stressors and job control: effects on event-related coping success and well-being. J Occup Organ Psych 78:237-252

Eshoj P, Jepsen JR, Nielsen CV (2001) Long-term sickness absence risk-indicators among occupationally active residents of a Danish county. Occup Med 51(5):347-353

Evans O, Steptoe A (2002) The contribution of gender-role orientation, work factors and home stressors to psychological well-being and sickness absence in male- and female-dominated occupational groups. Soc Sci Med 54:481-492

Folkman S, Lazarus RS (1980) An analysis of coping in a middle-aged community sample. J Health Soc Behav 21:219-239

Goffin RD (1993) A comparison of two new indices for the assessment of fit of structural equation models. Multivariate Behav Res 28:205-214

Hamilton S, Fagot BI (1988) Chronic stress and coping styles: a comparison of male and female undergraduates. J Pers Soc Psychol 55:819-823

Hensing G, Spak F, Alexanderson K, Allebeck P (1997) Sick leave among women and the role of psychiatric disorder. Scand J Soc Med 25:185-192

Hensing G, Alexanderson K, Allebeck P, Bjurulf P (1998) How to measure sickness absence? Literature review and suggestion of five basic measures. Scand J Soc Med 26:133-144

Heppner PP (1988) The problem solving inventory manual. Consulting psychologists press, Palo Alto

Heppner PP, Baker P (1997) Applications of the problem solving inventory. Meas Eval Couns Dev 9:229-241

Holahan Charles J., Moos Rudolf H., Schaefer Jeanne A (1996) Coping, stress resistance, and growth: conceptualizing adaptive functioning. In: Zeidner ME, Endler NSE (eds) Handbook of coping: theory, research, applications. Wiley, Oxford, pp 24-43

Huizink AC, de Medina PG, Mulder EJ, Visser GH, Buitelaar JK (2002) Coping in normal pregnancy. Ann Behav Med 24:132-140
Isacsson A, Hanson BS, Janzon L, Kugelberg G (1992) The epidemiology of sick leave in an urban population in Malmo, Sweden. Scand J Soc Med 20:234-239

Jeurissen T, Nyklicek I (2001) Testing the vitamin model of job stress in Dutch health care workers. Work Stress 15:254-264

Johansson G, Lundberg I (2004) Adjustment latitude and attendance requirements as determinants of sickness absence or attendance. Empirical tests of the illness flexibility model. Soc Sci Med 58:1857-1868

Kahn RL, Wolfe DM, Quinn RP, Snoek D, Rosenthal RA (1964) Organization stress: studies in role conflict and ambiguity. Wiley, New York

Kaplan EL, Meier P (1958) Nonparametric estimation from incomplete observations. J Am Stat Ass 53:457-481

Kohn PM (1996) On coping adaptively with daily hassles. In: Zeidner M, Endler NS (eds) Handbook of coping: theory, research, applications. Wiley, New York, pp 181-201

Kristensen TS (1991) Sickness absence and work strain among Danish slaughterhouse workers: an analysis of absence from work regarded as coping behaviour. Soc Sci Med 32:15-27

Landstad B, Vinberg S, Ivergard T, Gelin G, Ekholm J (2001) Change in pattern of absenteeism as a result of workplace intervention for personnel support. Ergonomics 44(1):63-81

Lazarus RS (1993) Coping theory and research: past, present, and future. Psychosom Med 55:234-247

Lazarus RS, Folkman S (1984) Stress, appraisal and coping. Springer, New York

Marmot M, Feeney A, Shipley M, North F, Syme SL (1995) Sickness absence as a measure of health status and functioning: from the UK Whitehall II study. J Epidemiol Community Health 49:124130

Mastekaasa A (2000) Parenthood, gender and sickness absence. Soc Sci Med 50:1827-1842

McWilliams LA, Cox BJ, Enns MW (2003) Use of the coping inventory for stressful situations in a clinically depressed sample: factor structure, personality correlates, and prediction of distress. J Clin Psychol 59(12):1371-1385

Moos RH, Holahan CJ (2003) Dispositional and contextual perspectives on coping: towards an integrative framework. J Clin Psychol 59(12):1387-1403

Norberg AL, Lindblad F, Boman KK (2005) Coping strategies in parents of children with cancer. Soc Sci Med 60(5):965-975

Nunnally JC (1967) Psychometric theory, 1st edn. McGraw-Hill, New York

Nunnally JC (1978) Psychometric theory, 1st edn. McGraw-Hill, New York

Nunnaly JC, Bernstein IH (1994). Psychometric theory, 3rd edn. MacGraw-Hill, New York

Parker JD, Endler NS (1992) Coping with coping assessment: a critical review. Eur J Pers 6:321-344

Pearlin LI, Schooler C (1978) The structure of coping. J Health Soc Behav 19:2-21

Peterson RA (1994) A meta-analysis of Cronbach's alpha. J Cons Res 21:381-391

Penley JA, Tomaka J, Wiebe JS (2002) The association of coping to physical and psychological health outcomes: a meta-analytic review. J Behav Med 25:551-603

Pisarski A, Bohle P, Callan VJ (1998) Effects of coping strategies, social support and work-nonwork conflict on shift worker's health. Scand J Work Env Health 24(Suppl 3):141-145

Roesch SC,.Weiner B (2001) A meta-analytic review of coping with illness: do causal attributions matter? J Psychosom Res 50:205-219

Rosvold EO, Bjertness E (2001) Physicians who do not take sick leave: hazardous heroes? Scand J Public Health 29:71-75

Sandanger I, Nygard JF, Brage S, Tellnes G (2000) Relation between health problems and sickness absence: gender and age differ- 
ences-a comparison of low-back pain, psychiatric disorders, and injuries. Scand J Public Health 28:244-252

Schaufeli WB, Van Dierendonck D (1992) De betrouwbaarheid en validiteit van de Utrechtse Coping Lijst: Een longitudinaal onderzoek bij schoolverlaters (The reliability and validity of the Utrecht Coping List. A longitudinal study among school-leavers). Gedrag Gezond 20:38-45

Schreurs PJG, Van De Willige G, Brosschot JF, Tellegen B, Graus GMH. (1993) De Utrechtse Coping Lijst: UCL. Swets \& Zeitlinger, Lisse

Somerfield MR, McCrae RR (2000) Stress and coping research: methodological challenges, theoretical advances, and clinical applications. Am Psychol 55:620-625

Stansfeld SA, Rael GS, Head J, Shipley M, Marmot M (1997) Social support and psychiatric sickness absence: a prospective study of British civil servants. Psychol Med 27(1):35-48

Stanton AL, Danoff-Burg S, Sworowski LA, Collins CA, Branstetter AD, Rodriguez-Hanley A et al. (2002a) Randomized, controlled trial of written emotional expression and benefit finding in breast cancer patients. J Clin Oncol 20:4160-4168
Stanton AL, Parsa A, Austenfeld JL (2002b) The adaptive potential of coping through emotional approach. In: Snyder CR, Lopez SJ (eds) Handbook of positive psychology. Oxford University Press, New York, pp 148-158

Tamres LK, Janicki D, Helgeson V (2002) Sex differences in coping behavior: a meta-analytic review and an examination of relative coping. Pers Soc Psychol Rev 6(1):2-30

Terry DJ, Callan VJ, Sartori G (1996) Employee adjustment to an organizational merger: stress, coping and intergroup differences. Stress Med 12:105-122

Vahtera J, Pentti J, Uutela A (1996) The effect of objective job demands on registered sickness absence spells: do personal, social and job-related resources act as moderators? Work Stress 10:286308

Voss M, Floderus B, Diderichsen F (2001) Physical, psychosocial, and organisational factors relative to sickness absence: a study based on Sweden post. Occup Environ Med 58(3):481-486

Whitaker SC (2001) The management of sickness absence. Occup Environ Med 58:420-424 